\title{
CTTITEM: SAS macro and SPSS syntax for classical item analysis
}

\author{
Pui-Wa Lei and Qiong Wu \\ Pennsylvania State University, University Park, Pennsylvania
}

\begin{abstract}
This article describes the functions of a SAS macro and an SPSS syntax that produce common statistics for conventional item analysis including Cronbach's alpha, item difficulty index ( $p$-value or item mean), and item discrimination indices (D-index, point biserial and biserial correlations for dichotomous items and item-total correlation for polytomous items). These programs represent an improvement over the existing SAS and SPSS item analysis routines in terms of completeness and user-friendliness. To promote routine evaluations of item qualities in instrument development of any scale, the programs are available at no charge for interested users. The program codes along with a brief user's manual that contains instructions and examples are downloadable from suen.ed.psu.edu/ ppwlei/plei.htm.
\end{abstract}

In any test or instrument development, newly written items are necessarily tried out or pilot tested before they can be used to collect information. Qualities of individual items such as whether they are functioning the way as intended have to be evaluated. Items deemed problematic are often revised or eliminated from the final form. Item statistics based on tryout data are informative for such decisions.

Conventional item analysis typically includes such tasks as gauging difficulty (prevalence of correct responses) and discrimination (ability to differentiate respondents on the trait being measured) for each item as well as estimating score reliability and distribution for the set of items to be used as a whole. Results of an item analysis can help determine the minimum number of items needed for a desired level of score reliability or measurement accuracy. The common statistics used by the classical item and test analysts for dichotomously scored (e.g., multiplechoice or true/false items) and polytomously scored (e.g., Likert-type scale or essay questions) items are produced by the customized SAS macro and SPSS syntax. These programs are customized to generate user-friendly outputs that are similar to those produced by a specialized item analysis software program such as ITEMAN (Assessment Systems Corporation, 1989) but without the accompanied cost of commercial programs. They represent an update or improvement over the existing routines within SAS and SPSS as well as supporting materials available from the Web.

Currently, there is not a SAS built-in item analysis routine though an "item" macro that performs item analysis for multiple-choice items is available from the Web (Sample 478, 1992). However, the SAS "item" macro does not provide the index of discrimination (D-index) and it is not clear how ties are treated when forming the high and low scoring groups for distractor analyses. Although SPSS has a "reliability" routine for item analysis, its use is limited. Without additional customization, scoring is tedious in current versions of SPSS. Moreover, the current "reliability" routine of SPSS does not produce the D-index and conditional option distributions for distractor analysis. Levesque (2003) provided an SPSS syntax that is supposed to perform item analysis of multiple-choice items as described in the SPSS white paper (1998). However, the syntax produces some error messages and does not generate the D-index when it is run in SPSS 13, due perhaps to some incompatibility between different SPSS versions. Moreover, neither program performs item analysis for polytomously scored items. Instructions on how to use these programs as well as documentations about how some of the statistics are computed are also not very detailed.

We created the CTTITEM SAS macro and SPSS syntax to overcome these shortcomings. Specifically, item analyses are performed for not only dichotomous items but also polytomous items. In addition, the D-index is calculated for dichotomous items. The programs are also made more user-friendly by reducing the number of required input modifications and providing brief instructional manuals. Details of the program functions are described below.

\section{CTTITEM: A SAS Macro}

CTTITEM is a SAS macro that performs classical item analysis of dichotomously scored items, such as those of the multiple-choice or true-false format; as well as polytomously scored items, like short-answer or Likert-type questions. Being a SAS macro, CTTITEM can be run on any platform with which SAS is compatible, and the job

P.-W. Lei, puiwa@psu.edu 
size is limited only by your computer's memory allocation to SAS.

CTTITEM scores option or category responses based on the desired type of scoring (dichotomous or polytomous). For dichotomous items, the macro reads in option responses and scores the items as right or wrong with the answer keys provided. For polytomous items, on the other hand, it reads in category responses and reverses score categories for negatively stated questions as specified by the user. Descriptive statistics for total score such as mean, standard deviation, quartiles, skewness, and kurtosis, as well as Cronbach's alpha (Cronbach, 1951) and standard error of measurement (SEM) for the test are reported.

Moreover, CTTITEM calculates item difficulty ( $p$-value for dichotomous items and item mean for polytomous items), various item discrimination indices (corrected and uncorrected point-biserial correlation, biserial correlation, and index of discrimination for dichotomous items; corrected and uncorrected item-total correlation for polytomous items; see Crocker \& Algina, 1986, and Ebel \& Frisbie, 1991, for detailed discussions of these item statistics), and Cronbach's alpha when the item in question is deleted. For dichotomous items, index of discrimination is computed based on Kelley (1939)'s 27\% rule in classifying the high and low scoring groups. When there are ties at the cut scores, they are included in their corresponding groups. This grouping is also used to tabulate conditional relative frequency distributions of option responses (by high and low groups expressed in percentages) for option analysis. For polytomous items, marginal distributions of item categories are provided. Data sets for scored item responses as well as item statistics are created and can be output for additional analysis.

The data to be analyzed should have the following format:

1st row: "ID" in the 1st column, followed by item variable names (this row is optional and must be bypassed with the "firstobs $=2$ " option in the data step when the data set is read in if it is present).

2nd row: uppercase "NCAT" in the 1st column, followed by the number of options/categories for each item.

3rd row: uppercase "KEY" in the 1st column, followed by the keys for objective items to be scored dichotomously, or for polytomous items, enter " 1 " for positively scored or " 0 " for reversely scored items (e.g., all 1's when no reverse scoring is necessary).

4th to last row: subjects' id in the 1 st column, followed by their item responses (note: use one row for each subject, one column for each item, and a "." for missing response).

To call the CTTITEM macro, simply copy the entire macro text from "\%MACRO..." to "\%MEND..." onto the SAS program editor, type "\%CTTITEM(-, -, -, -, -);" with the dashes replaced by the proper parameter values, and then submit. Inside the parentheses are parameter values in the following order: data set name to be analyzed, the nonnumerical prefix of the item variables, sample size, number of items, and the type of scoring desired (enter
"1" for dichotomous or "2" for polytomous items). An example including SAS data step commands used to read in an external data file is illustrated in the Appendix.

\section{SPSS Syntax}

A similar version of CTTITEM was also created in SPSS. For dichotomous items, it scores response options as right or wrong based on the answer keys provided by the user. For polytomous items, it reverse-scores negatively stated items so that all items have a consistent scoring scheme. It produces score distribution statistics (mean, standard deviation, maximum, minimum, skewness and kurtosis), Cronbach's alpha, item difficulty index (proportion correct for dichotomous items and item mean for polytomous items), item discrimination indices (D-index and corrected point-biserial correlation for dichotomous items and corrected item-total correlation for polytomous items), score frequency tables for polytomous items, and distractor analysis results for multiple-choice items (frequency distribution of options endorsed by low- and highscoring groups). The scoring groups are formed based on the same scheme as that used in the SAS macro. Two separate SPSS syntax files are available, one for dichotomous items (CTTITEM_D.sps) and the other for polytomous items (CTTITEM_P.sps). The following instructions on how to use the syntax apply to both cases.

Input data. Input data should be in text format. The first line should contain the correct options for dichotomous items or the number of score categories for polytomous items (the program assumes that your responses/ scores for the polytomous items start from 1 with an interval of 1). For dichotomous items, item response data start from the second line with each line representing one case. For polytomous items, the second line instructs the program whether to reversely score the items (0) or not (1). Examinee scores start from the third line for polytomous items. Use a space for a missing value. Missing value will be treated as incorrect for the calculation of item and test statistics for dichotomous items. Listwise deletion is used for missing values of polytomous items. The program will score dichotomous items as right (coded as " 1 ") or wrong (coded as " 0 ") based on the answer keys provided by the user. For polytomous items, the program will reversely score the items instructed by the user (i.e., "0"s on the second line of the data file). For example, if the user would like to have high scores to represent high levels of the trait being measured, then a " 1 " should be entered on the second line of the data file for all positively stated items and a " 0 " for all negatively stated items.

Syntax. A few changes need to be made to the syntax to fit the current data structure. They are file locations and variable names and have been highlighted with instructions in the syntax files (see the brief manual included in the package for additional details).

Output. The first part of the output includes the statistics of the total score distribution. The reliability analysis results that follow include Cronbach's alpha, summary statistics for interitem correlations, and item-total statis- 
tics. The "corrected item-total correlation" is commonly called the corrected point-biserial correlation. $P$-value and D-index are then listed for every item in its original order. For polytomous items, $p$-value is replaced by item mean, and D-index does not apply. If the data are dichotomously scored, frequencies of options endorsed by the low- and high- scoring groups (distractor analysis) will be displayed. If the data are polytomously scored, score point frequencies for every item will be provided. Scored item responses, total scores, as well as item $p$-value and D-index are saved in separate data sets for later use.

\section{Program Availability}

CTTITEM was developed using SAS/IML, version 9.1 and is compatible with earlier versions of SAS/IML (e.g., SAS Version 8.1). The SPSS version was developed with the SPSS (Version 13.0) matrix language and is also compatible with older versions (e.g., SPSS Version 11.5). Syntax files of the SAS and SPSS program codes, brief manuals, example data and output files are available for download, free-of-charge, from the first author's Web site: suen.ed.psu.edu/ pwlei/plei.htm.

\section{AUTHOR NOTE}

Correspondence concerning this article should be addressed to P.-W. Lei, 230 Cedar Building, Pennsylvania State University, University Park, PA 16802 (e-mail: puiwa@psu.edu).

\section{REFERENCES}

ASSESSMENT SySTEMS CORPORATION (1989). User's manual for the ITEMAN conventional item analysis program, version 3.5 for Windows 3.x. St. Paul, MN: Author.

Crocker, L., \& Algina, J. (1986). Introduction to classical and modern test theory. New York: Holt, Rinehart \& Winston.

Cronbach, L. J. (1951). Coefficient alpha and the internal structure of tests. Psychometrika, 16, 297-334.

EBEL, R. L., \& FRISBIE, D. A. (1991). Essentials of educational measurement (5th ed.) Englewood Cliffs, NJ: Prentice Hall.

KeLLEY, T. L. (1939). Selection of upper and lower groups for the validation of test items. Journal of Educational Psychology, 30, 17-24.

LEVESQue, R. (2003, January 27). Syntax for item analysis.SPS. Retrieved May 6, 2006, from www.spsstools.net/Syntax/ItemAnalysis/ SyntaxForItemAnalysis.txt.

SAMPle 478: PERForm ITEM ANALySis FOR MUltiple CHOICE TESTS (1992, June 26). Retrieved May 6, 2006, from support.sas.com/ctx/ samples/index.jsp?sid $=478 \&$ tab $=$ details.

SPSS WHITE PAPER: USING SPSS FOR ITEM ANALYSIS (1998). Retrieved May 6, 2006, from www.spsstools.net/Syntax/ItemAnalysis/ UsingSPSSforItemAnalysis.pdf. 
APPENDIX

SAS Example for a Hypothetical Test With 15 Multiple-Choice Items Taken by 100 Examinees

The sample data set "c: Imcitem.dat" contains:

$\begin{array}{lrrrrrrrrrrrrrrr}\text { ID } & 1 & 2 & 3 & 4 & 5 & 6 & 7 & 8 & 9 & 10 & 11 & 12 & 13 & 14 & 15 \\ \text { NCAT } & 4 & 4 & 4 & 4 & 4 & 4 & 4 & 4 & 4 & 4 & 4 & 4 & 4 & 4 & 4 \\ \text { KEY } & 4 & 3 & 1 & 1 & 2 & 4 & 2 & 1 & 3 & 1 & 1 & 2 & 3 & 2 & 1 \\ 1 & 4 & 3 & 1 & 2 & 2 & 2 & 4 & 1 & 3 & 1 & 3 & 2 & 3 & 2 & 1 \\ 2 & 4 & 3 & 1 & 1 & 2 & 4 & 2 & 1 & 3 & 1 & 1 & 2 & 3 & 2 & 1 \\ 3 & 4 & 3 & 1 & 1 & 2 & 4 & 2 & 1 & 2 & 1 & 1 & 2 & 3 & 2 & 1 \\ 4 & 4 & 3 & 1 & 1 & 2 & 1 & 2 & 1 & 3 & 1 & 3 & 2 & 2 & 2 & 1 \\ 5 & 4 & 3 & 1 & 3 & 2 & 2 & 1 & 1 & 2 & 2 & 1 & 2 & 3 & 2 & 1\end{array}$

The SAS commands used to read in the external data file and invoke the CTTITEM macro:

DATA ONE;

INFILE 'C: $\mid$ MCITEM.DAT' FIRSTOBS $=2$;

INPUT ID \$ MC1-MC15;

RUN;

\%CTTITEM(ONE,MC,100,15,1);

RUN;

The output generated by SAS:

TEST STATISTICS FOR MC

TOTAL_MC

$\mathrm{N}$

STD $\quad 2.43$

MIN $\quad 5.00$

Q1 11.00

MEDIAN $\quad 13.00$

Q3 $\quad 15.00$

MAX $\quad 15.00$

SKEWNESS $\quad-1.05$

KURTOSIS $\quad 0.57$

ALPHA $\quad 0.73$

SEM $\quad 1.27$

/item statistics/

$\begin{array}{lrrrrrr} & \text { P_VALUE } & \text { POINT_BISERIAL } & \text { BISERIAL } & \text { CORRECTED_PTBIS } & \text { D_INDEX } & \text { ALPHA_WITHOUT_ITEM } \\ \text { WRMC1 } & 0.96 & 0.41 & 0.93 & 0.34 & 0.10 & 0.72 \\ \text { WRMC2 } & 0.99 & 0.27 & 1.02 & 0.23 & 0.03 & 0.73 \\ \text { WRMC3 } & 0.97 & 0.38 & 0.96 & 0.32 & 0.10 & 0.72 \\ \text { WRMC4 } & 0.90 & 0.25 & 0.42 & 0.13 & 0.21 & 0.73 \\ \text { WRMC5 } & 0.85 & 0.48 & 0.74 & 0.36 & 0.34 & 0.71 \\ \text { WRMC6 } & 0.79 & 0.54 & 0.77 & 0.41 & 0.48 & 0.70 \\ \text { WRMC7 } & 0.83 & 0.51 & 0.75 & 0.38 & 0.41 & 0.71 \\ \text { WRMC8 } & 0.92 & 0.44 & 0.80 & 0.34 & 0.21 & 0.71 \\ \text { WRMC9 } & 0.71 & 0.52 & 0.69 & 0.36 & 0.59 & 0.71 \\ \text { WRMC10 } & 0.73 & 0.42 & 0.56 & 0.25 & 0.48 & 0.72 \\ \text { WRMC11 } & 0.65 & 0.51 & 0.65 & 0.34 & 0.72 & 0.71 \\ \text { WRMC12 } & 0.88 & 0.53 & 0.87 & 0.43 & 0.41 & 0.70 \\ \text { WRMC13 } & 0.78 & 0.52 & 0.73 & 0.38 & 0.45 & 0.71 \\ \text { WRMC14 } & 0.83 & 0.61 & 0.90 & 0.49 & 0.55 & 0.69 \\ \text { WRMC15 } & 0.80 & 0.48 & 0.69 & 0.34 & 0.48 & 0.71\end{array}$

/Relative \% frequency distributions by high and low groups for each item. "“* indicates the item key./ IRMC1

\begin{tabular}{lrrrrr}
\multicolumn{1}{l}{ LOW $27 \%$} & $\mathrm{~N}$ & 1 & 2 & 3 & $*$ \\
HI $27 \%$ & 29 & 3 & 7 & 0 & 90 \\
\multicolumn{1}{r}{ IRMC2 } & 29 & 0 & 0 & 0 & 100 \\
LOW $27 \%$ & $\mathrm{~N}$ & 1 & 2 & $*$ & 4 \\
HI $27 \%$ & 29 & 3 & 0 & 97 & 0 \\
& 29 & 0 & 0 & 100 & 0
\end{tabular}

(Similar outputs are omitted.) 